\title{
Eliminating the Detrimental Effects of Conductive Agents in Sulfide-Based Solid- State Batteries
}

Sixu Deng, ${ }^{\dagger}$ Yipeng Sun, ${ }^{\dagger}$ Xia Li, ${ }^{\dagger}$ Zhouhong Ren, ${ }^{\dagger}$ Jianwen Liang, ${ }^{\dagger}$ Kieran Doyle-Davis, ${ }^{\dagger}$ Jianneng Liang, ${ }^{\dagger}$ Weihan Li, ${ }^{\dagger}$ Mohammad Norouzi Banis, ${ }^{\dagger}$ Qian Sun, ${ }^{\dagger}$ Ruying Li, Yongfeng Hu,§ Huan Huang, ${ }^{\perp}$ Li Zhang," Shigang Lu," Jun Luo,* and Xueliang Sun*,†

${ }^{\dagger}$ Department of Mechanical and Materials Engineering, University of Western Ontario, London, Ontario N6A 5B9, Canada

$\$$ Center for Electron Microscopy and Tianjin Key Lab of Advanced Functional Porous Materials, Institute for New Energy Materials and Low-Carbon Technologies, School of Materials Science and Engineering, Tianjin University of Technology, Tianjin 300384, China

$\S$ Canadian Light Source, 44 Innovation Boulevard, Saskatoon, Saskatchewan S7N 2V3, Canada

" China Automotive Battery Research Institute Co. Ltd, Fifth Floor, No. 43, Mining Building, North Sanhuan Middle Road, Beijing 100088, China

${ }^{\perp}$ Glabat Solid-State Battery Inc., 700 Collip Circle, London, Ontario N6G 4X8, Canada

\section{Corresponding Author:}

*E-mail: xsun@eng.uwo.ca (Xueliang Sun) 


\section{Experimental Section}

Preparation of PEDOT modified CNTs and NMC811: The poly(3,4ethylenedioxythiophene) (PEDOT) was deposited on the surface of carbon nanotubes (CNTs) and $\mathrm{LiNi}_{0.8} \mathrm{Mn}_{0.1} \mathrm{Co}_{0.1} \mathrm{O}_{2}$ (NMC811) powders via molecular layer deposition (MLD) using molybdenum chloride $\left(\mathrm{MoCl}_{5}\right)$ and 3,4-ethylenedioxythiophene (EDOT) as precursors in an Arradiance GEMstar-8 system. The MLD machine was connected with the argon-filled glove box. The source temperatures for both $\mathrm{MoCl}_{5}$ and EDOT were $80{ }^{\circ} \mathrm{C}$. The deposition temperature for PEDOT was $150{ }^{\circ} \mathrm{C}$. During one MLD cycle, $\mathrm{MoCl}_{5}$ and EDOT were alternatively introduced into the reaction chamber with a pulse time of $5 \mathrm{~s}$, and the pulsing of each precursor was separated by a $60 \mathrm{~s}$ purge with $\mathrm{N}_{2}$.

Assembly of the tested batteries and electrochemical testing: In order to investigate the stability between carbon additives and sulfide electrolytes, the sandwiched model cells were fabricated by using the as-prepared cathode composites combining with commercial $\mathrm{Li}_{10} \mathrm{GeP}_{2} \mathrm{~S}_{12}$ (LGPS, 99.95\%, MSE supplies, LLC) as electrolyte layer and indium foil (99.99\%, $\Phi 10 \mathrm{~mm}$, thickness $0.1 \mathrm{~mm})$ as anode. The detailed fabrication process is shown as follow. Firstly, $70 \mathrm{mg}$ LGPS was pressed at 2 ton with $10 \mathrm{~mm}$ diameter to form a pellet. The cathode composite power $(7 \mathrm{mg})$ was then uniformly spread onto the surface of the LGPS electrolyte and pressed under 3 tons of pressure to form a two-layer pellet. The as-prepared cathode composites include the pristine LGPS (7 mg), bare CNTs mixed with LGPS (1 mg CNTs and 6 mg LGPS), PEDOT protected CNTs mixed with LGPS (1 mg CNTs and 6 mg LGPS), bare CNTs (7 mg), and PEDOT 
protected CNTs $(7 \mathrm{mg})$. Finally, a piece of indium foil was put onto the other side of the LGPS pellet with a copper foil and pressed at 0.5 tons of pressure. The three-layered pellet was pressed between two stainless-steel rods as current collectors at the both positive and negative sides. The fabrication of all-solid-state batteries is similar to the process for the above model cells. The difference is the cathode layer was fabricated by the mixing of the as-prepared NMC811/LGPS/CNTs composites (10 mg with a weight ratio of 65:30:5). All the processes were performed in an argon-filled glove box. The galvanostatic charge/discharge characteristics were tested in the range of 2.1-3.8 V vs. $\mathrm{Li}^{+} / \mathrm{Li}-\mathrm{In}$, corresponding to $2.7-4.4 \mathrm{~V}$ vs. $\mathrm{Li}^{+} / \mathrm{Li}$, at room temperature using a multichannel battery tester (LAND CT-2001A, Wuhan Rambo Testing Equipment Co., Ltd.). Linear sweep voltammetry (LSV) was collected on a versatile multichannel potentiostat 3/Z (VMP3) using a scan rate of $0.05 \mathrm{mV} \mathrm{s}^{-1}$ for the model cells. Cyclic voltammograms $(\mathrm{CV})$ were collected on a versatile multichannel potentiostat $3 / \mathrm{Z}$ (VMP3) using a scan rate of $0.1 \mathrm{mV} \mathrm{s}^{-1}$ for the model cells and $0.05 \mathrm{mV} \mathrm{s}^{-1}$ for the allsolid-state batteries, respectively. Electrochemical impedance spectroscopy (EIS) was also performed on the versatile multichannel potentiostat 3/Z (VMP3) by applying an AC voltage of $10 \mathrm{mV}$ amplitude in the $7000 \mathrm{kHz}$ to $100 \mathrm{mHz}$ frequency range.

Characterizations: The morphology and microstructure of PEDOT modified CNTs and NMC811 cathodes were characterized using field emission scanning electron microscopy (FESEM, Hitachi S4800) equipped with an energy dispersive spectrometer (EDX), high-resolution transmission electron microscopy (HRTEM, JEOL 2010FEG) and aberration-corrected scanning transmission electron microscopy (STEM, Titan 
Cubed Themis G2 300) with elemental mapping images. The crystalline structure of as-prepared CNTs and NMC811 cathodes are conducted on a Bruker D8 Advance Diffractometer (Cu-Ka source, 40 kV, 40 mA). X-ray photoelectron spectroscopy (XPS) spectra of the as-prepared samples were measured with a monochromatic Al Ka source $(1486.6 \mathrm{eV})$ in a Kratos AXIS Nova Spectrometer. The Ar filled glovebox was connected with the XPS machine to avoid the exposure of air. Synchrotron-based Xray absorption near edge structure (XANES) measurements were carried out at the Canadian Light Source (CLS). S, P, Ni, Co, and Mn K-edge spectra were collected by fluorescence mode using $\operatorname{Si}(111)$ crystals on the Soft X-ray Microcharacterization Beamline (SXRMB). All of the samples were prepared in a vacuum environment prior to synchrotron measurements. To avoid air exposure, the samples were firstly covered with Mylar film in glovebox under Ar, and then transferred to the corresponding beamline chamber for further measurements. 

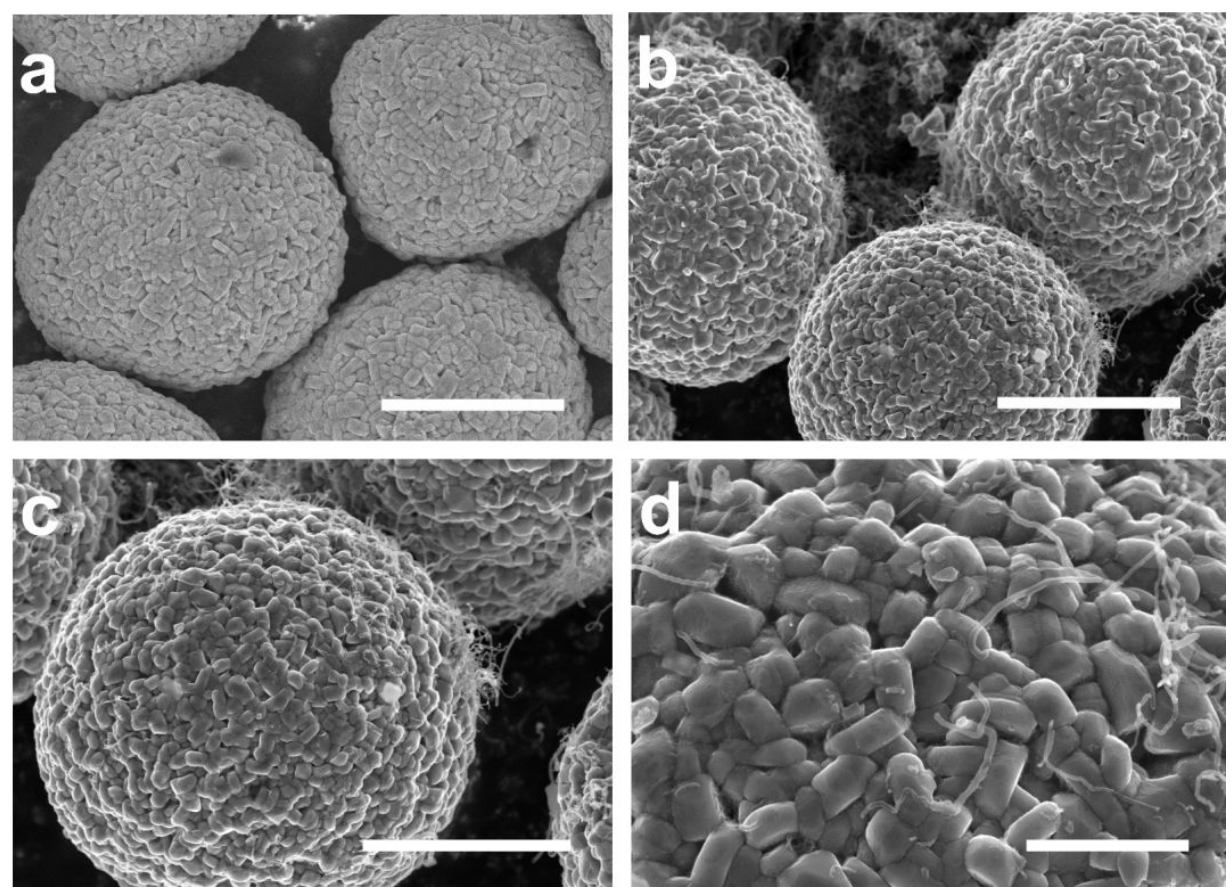

Figure S1. SEM images of a) bare NMC811; b-d) PEDOT modified NMC811/CNTs composites. Scale bars in the figures of a-c) $5 \mu \mathrm{m}$, and d) $1 \mu \mathrm{m}$. 


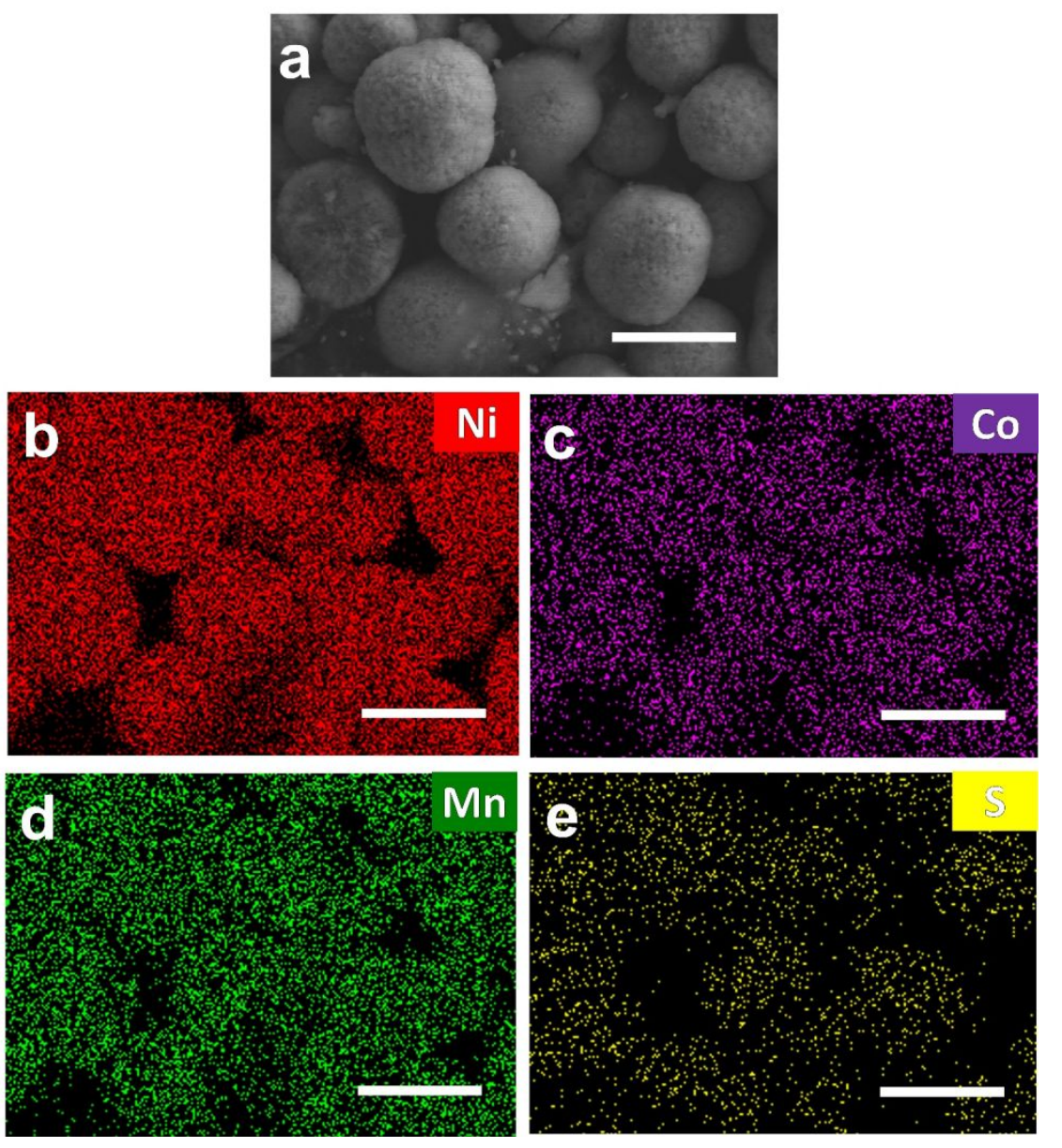

Figure S2. SEM-EDX mappings of PEDOT modified NMC811 cathode particles. b)

$\mathrm{Ni}, \mathrm{c}) \mathrm{Co}, \mathrm{d}) \mathrm{Mn}$, and e) S. Scale bars in the figures of $10 \mu \mathrm{m}$. 

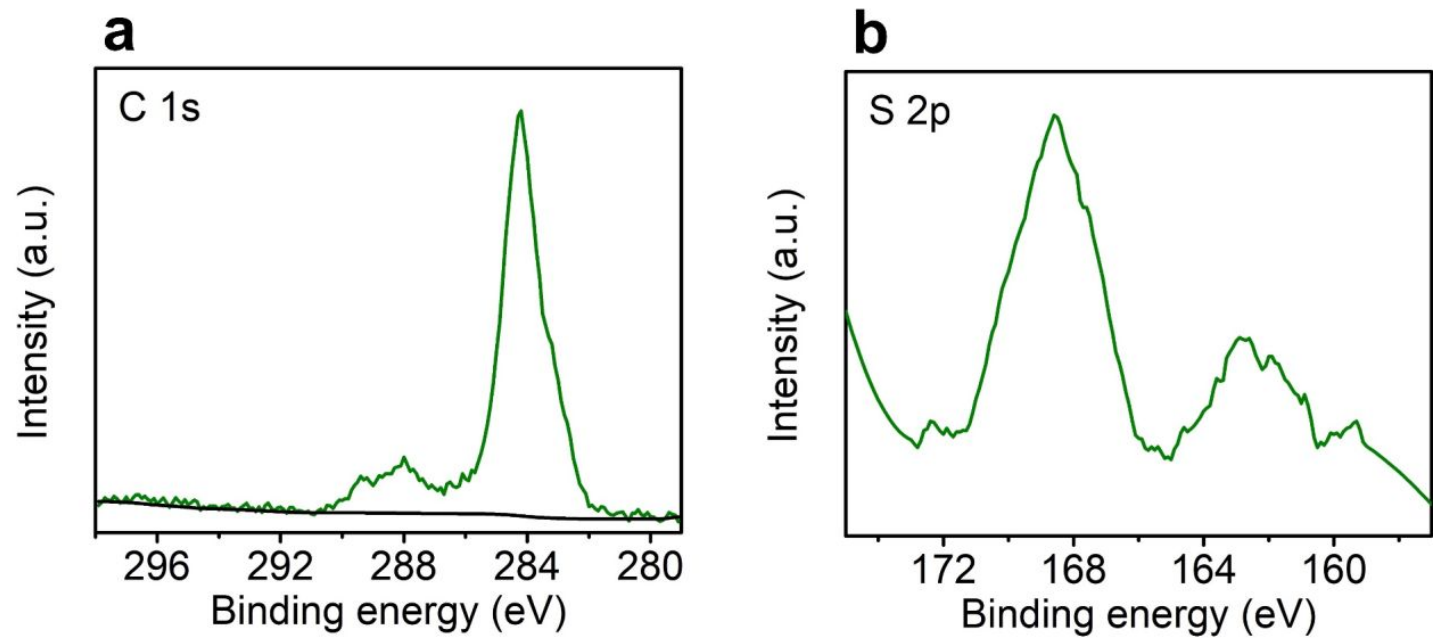

Figure S3. a) C 1s and b) S 2p XPS spectra of PEDOT modified NMC811. 

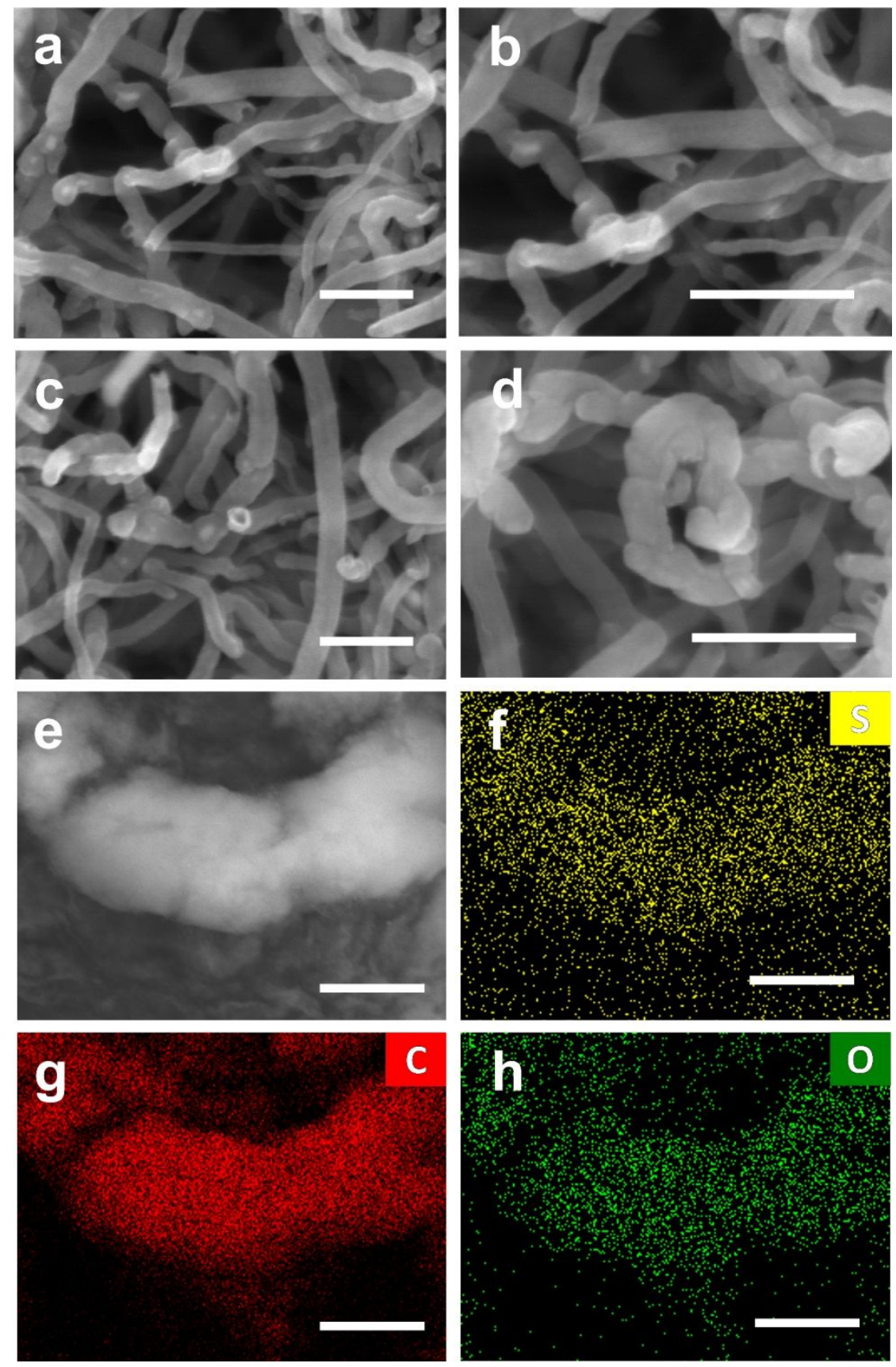

Figure S4. SEM images of a-b) bare CNTs; c-d) PEDOT modified CNTs; e-h) EDX mappings of PEDOT modified CNTs: f) S, g) C, and h) O. Scale bars in the figures of a and c) $150 \mathrm{~nm}, \mathrm{~b}$ and d) $200 \mathrm{~nm}, \mathrm{e}-\mathrm{h}) 15 \mu \mathrm{m}$. 


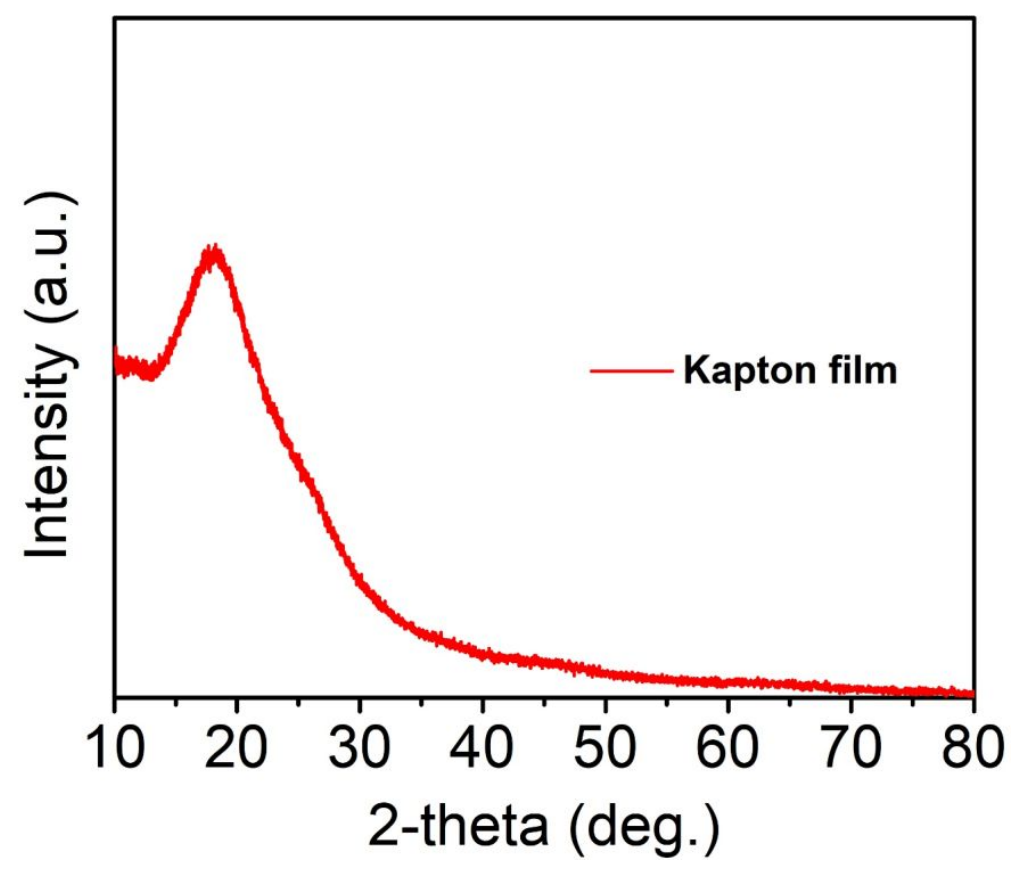

Figure S5. XRD pattern of Kapton film. 


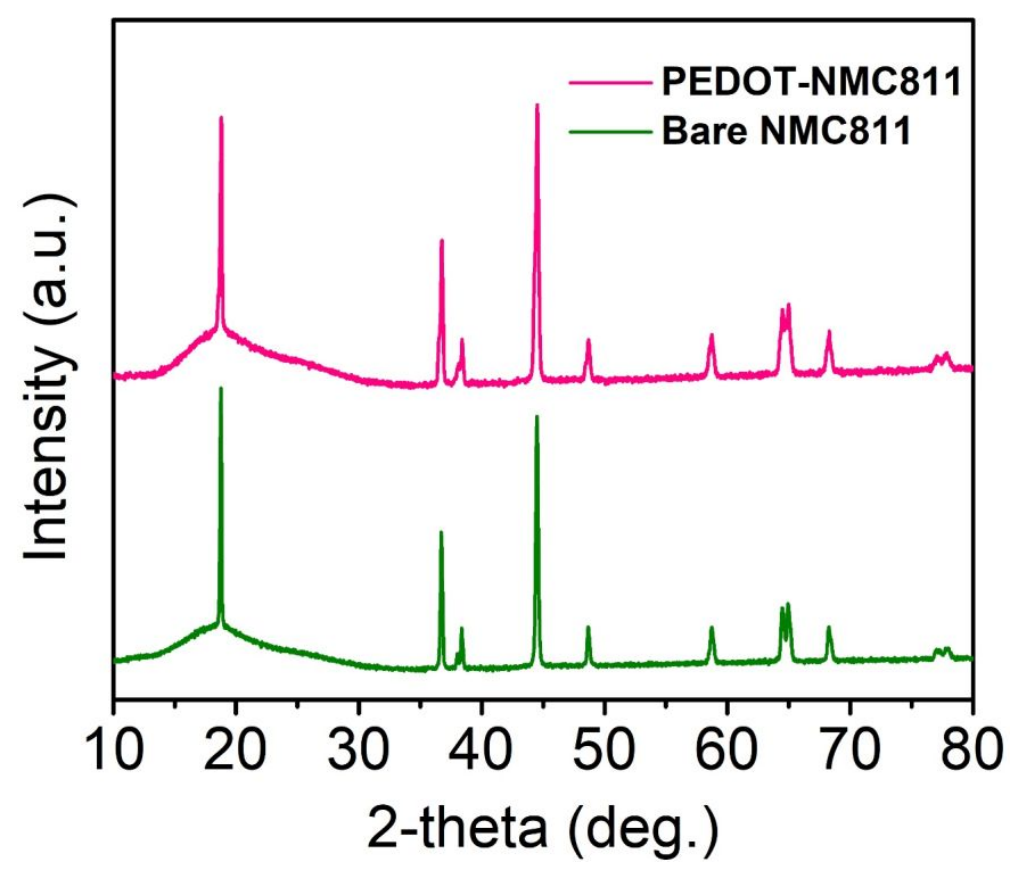

Figure S6. XRD patterns of the bare NMC811 and PEDOT modified NMC811. 


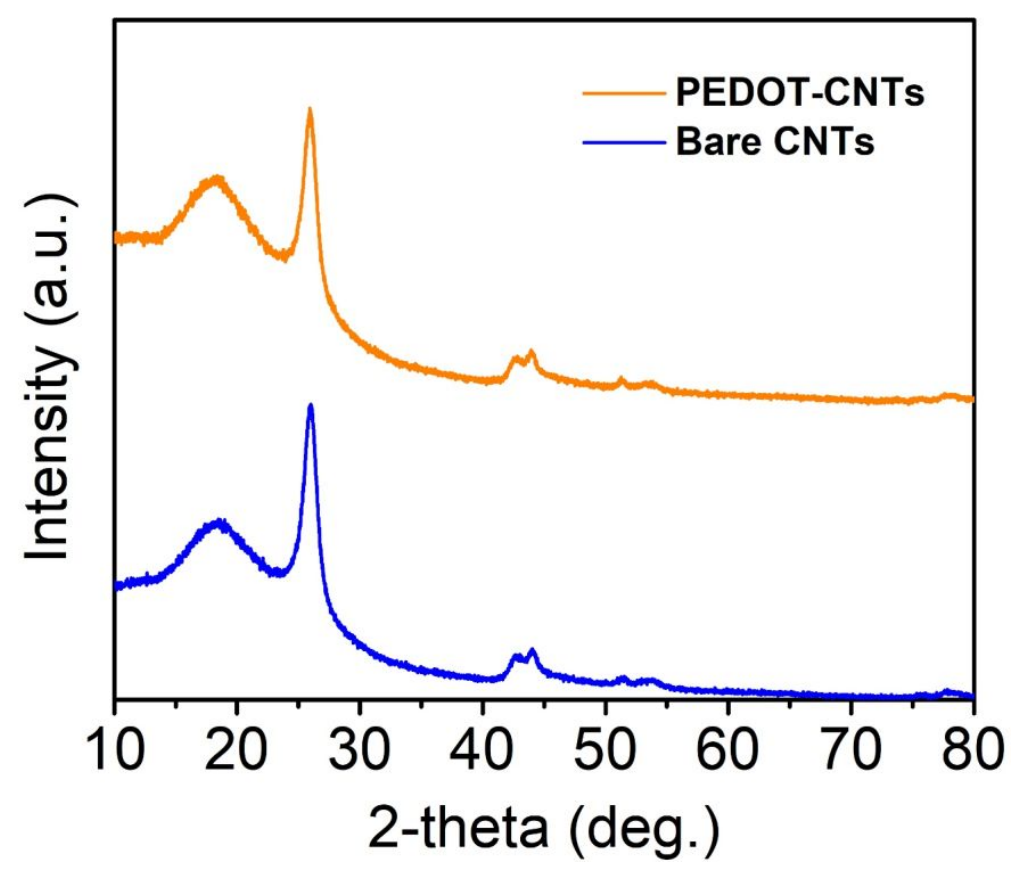

Figure S7. XRD patterns of the bare CNTs and PEDOT modified CNTs. 

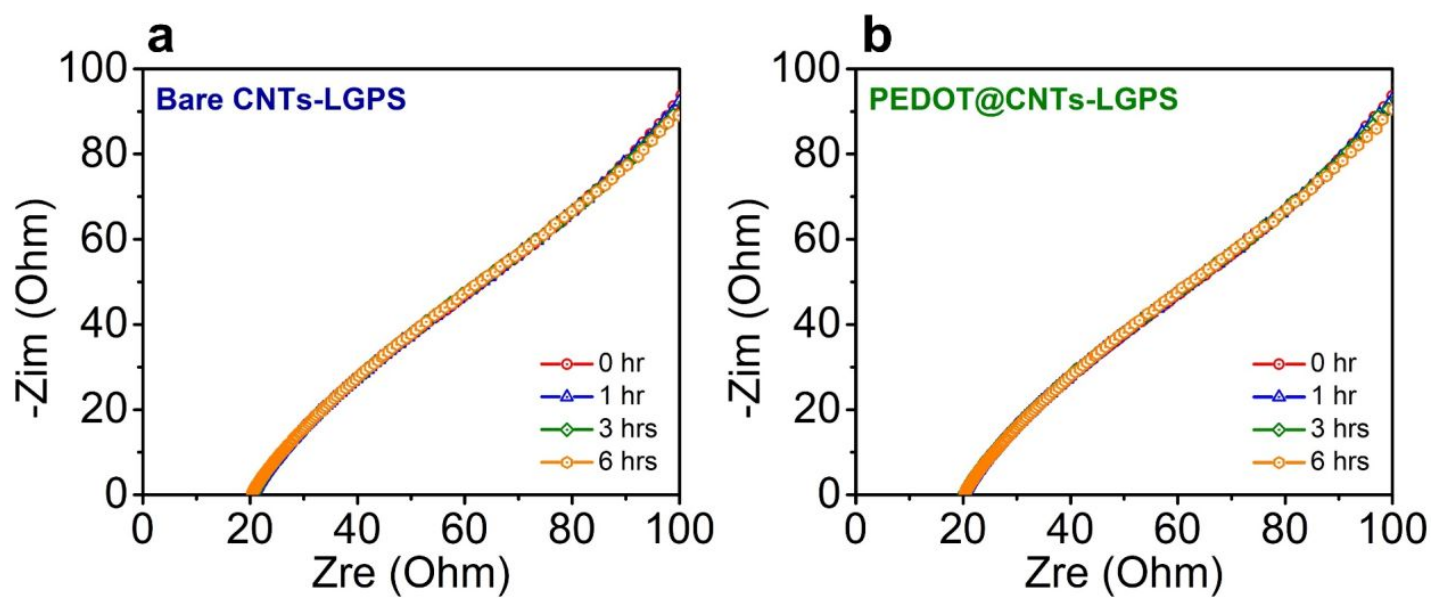

Figure S8. EIS plots of the Bare CNTs-LGPS and PEDOT@CNTs-LGPS cells assembled as shown in Figure 2a measured at a various resting time. 


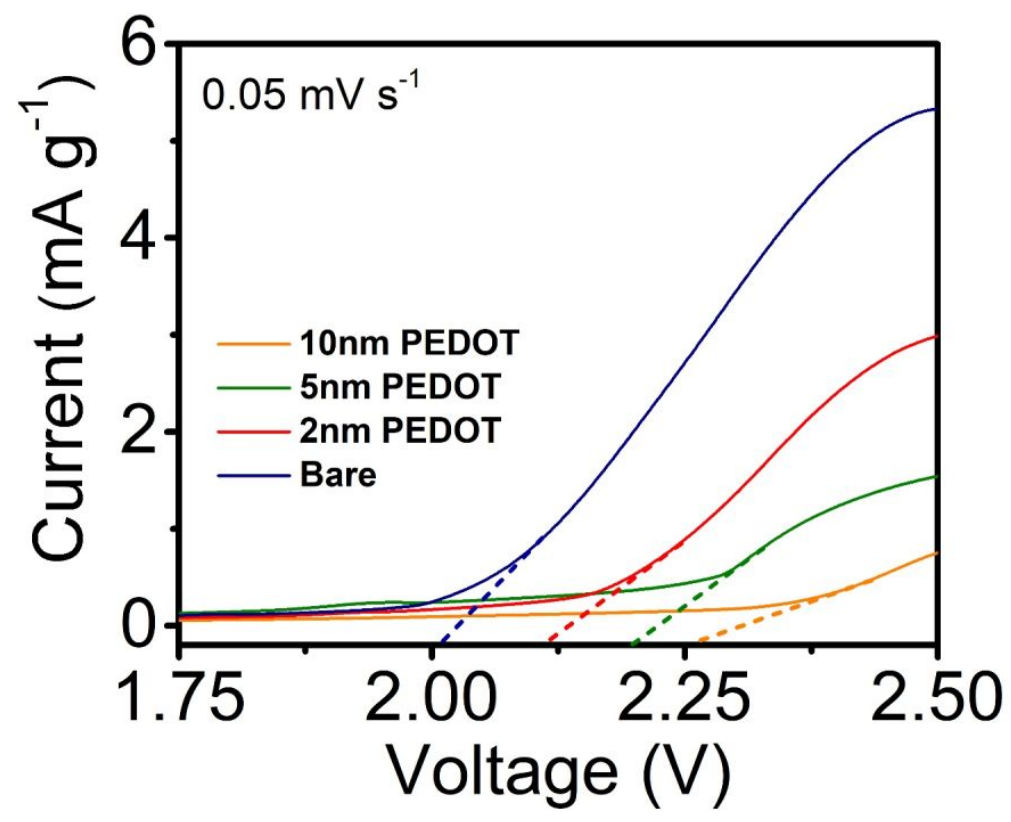

Figure S9. LSV profiles of the bare and different thickness PEDOT coated CNTs at $0.05 \mathrm{mV} \mathrm{s}^{-1}$. 


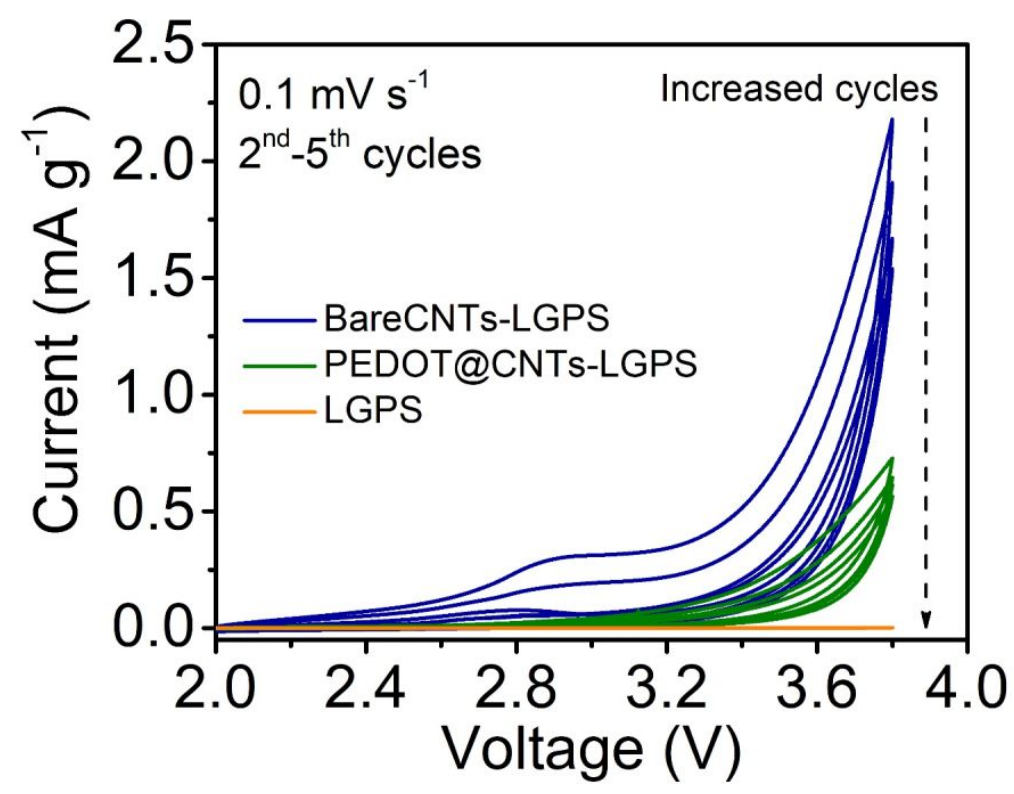

Figure S10. CV profiles of the different cathode composites as shown in Figure 2a during 2 nd to 5 th cycles. 
a

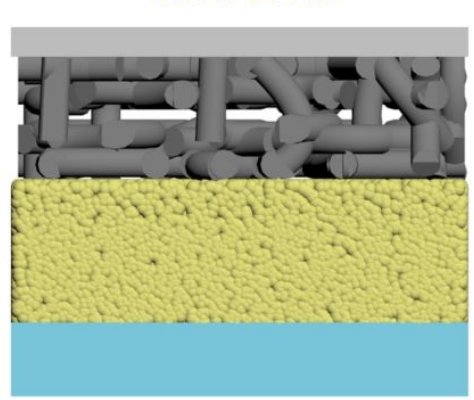

PEDOT@CNTs

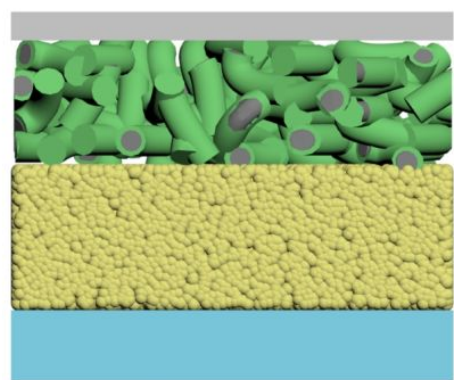

PEDOT coated CNTS
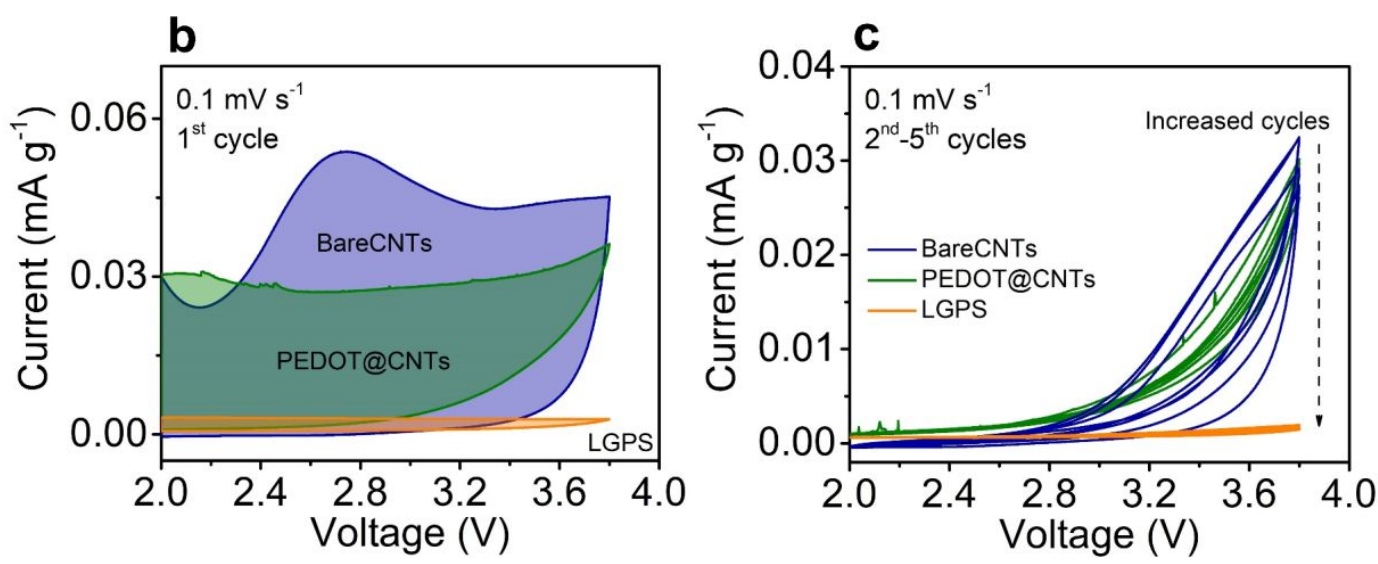

Figure S11. a) Schematic illustration of the designed model cells, b-c) CV profiles of the different cathode composites at $0.1 \mathrm{mV} \mathrm{s}^{-1}$ during 5 cycles. 


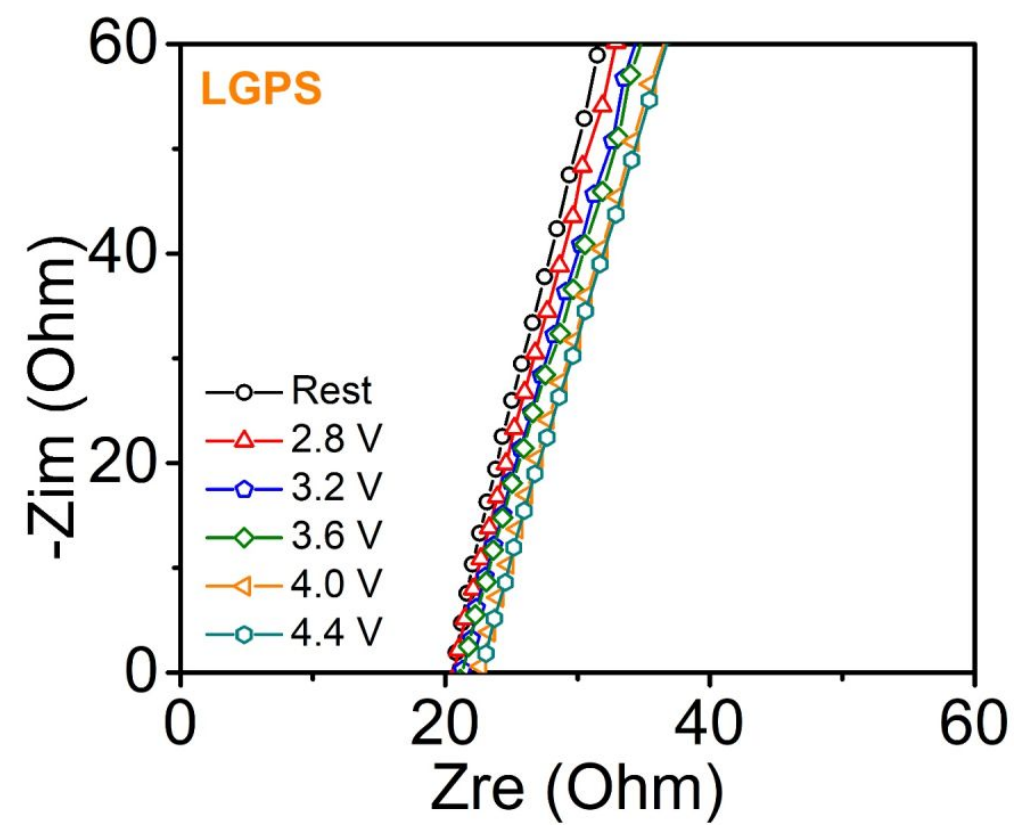

Figure S12. EIS spectra of the pristine LGPS at various constant voltages. 


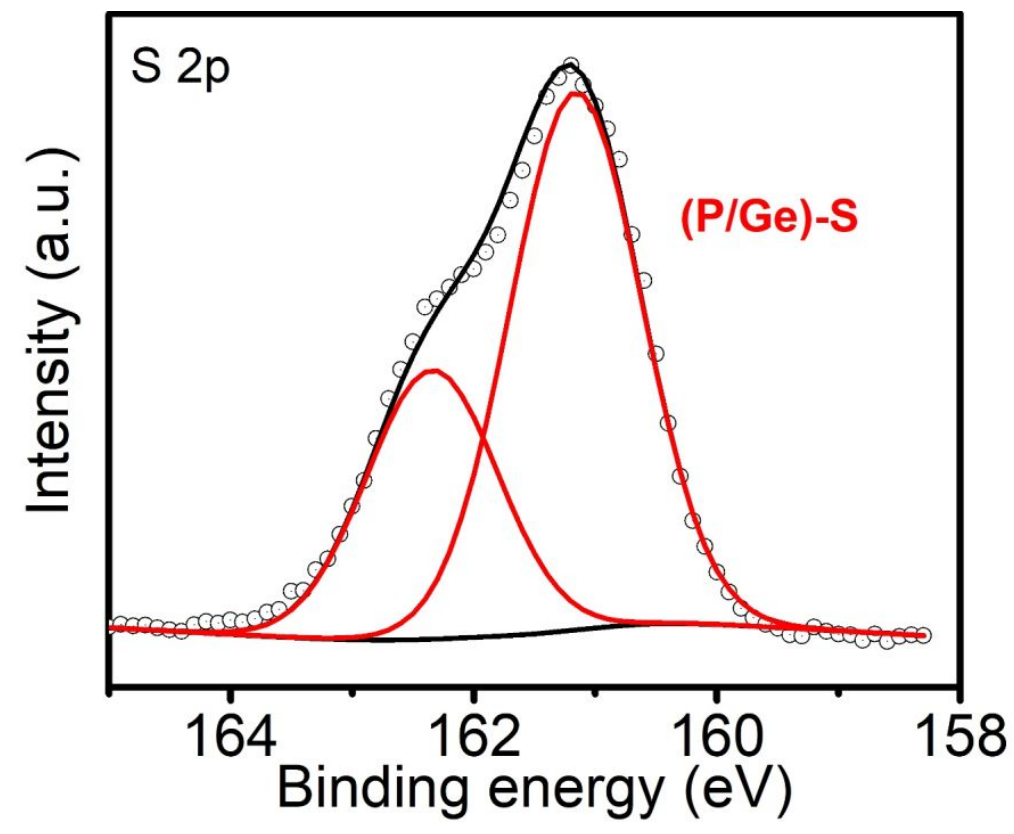

Figure S13. S $2 p$ spectrum of the pristine LGPS. 


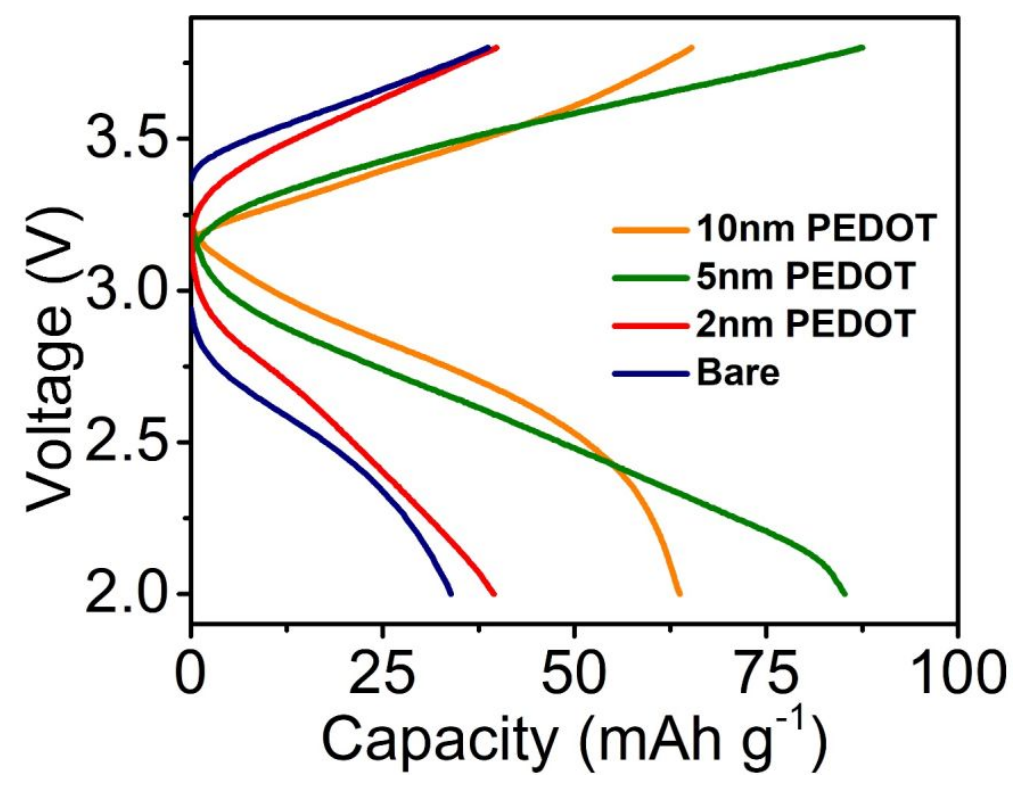

Figure S14. Charge/discharge curves of the 100 cycle at $0.05 \mathrm{C}$. 


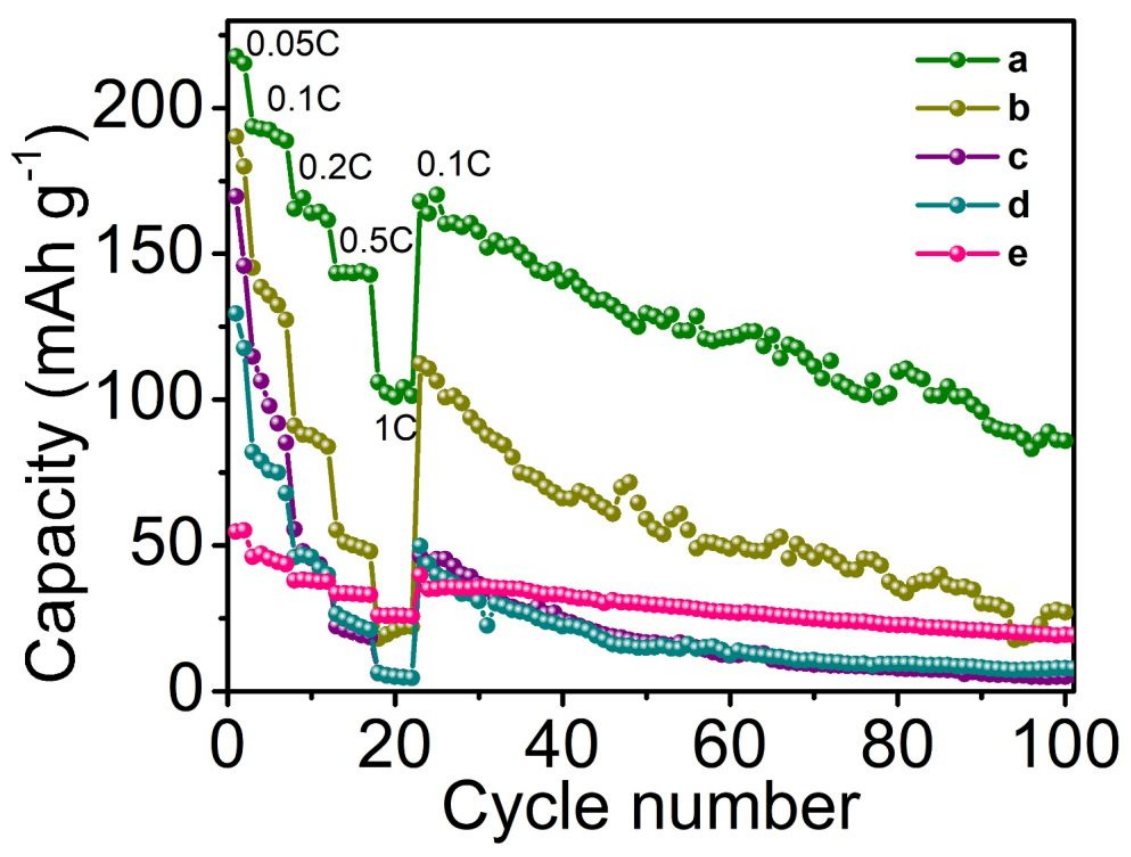

Figure S15. The rate and cycling stability of various coating strategies: a) PEDOT coating on both NMC811 and CNTs, b) $\mathrm{LiNbO}_{\mathrm{x}}$ coating on NMC811 and PEDOT coating on CNTs, c) PEDOT coating on CNTs with bare NMC811, d) PEDOT coating on NMC811 with bare CNTs, e) PEDOT coating on NMC811 without CNTs. 


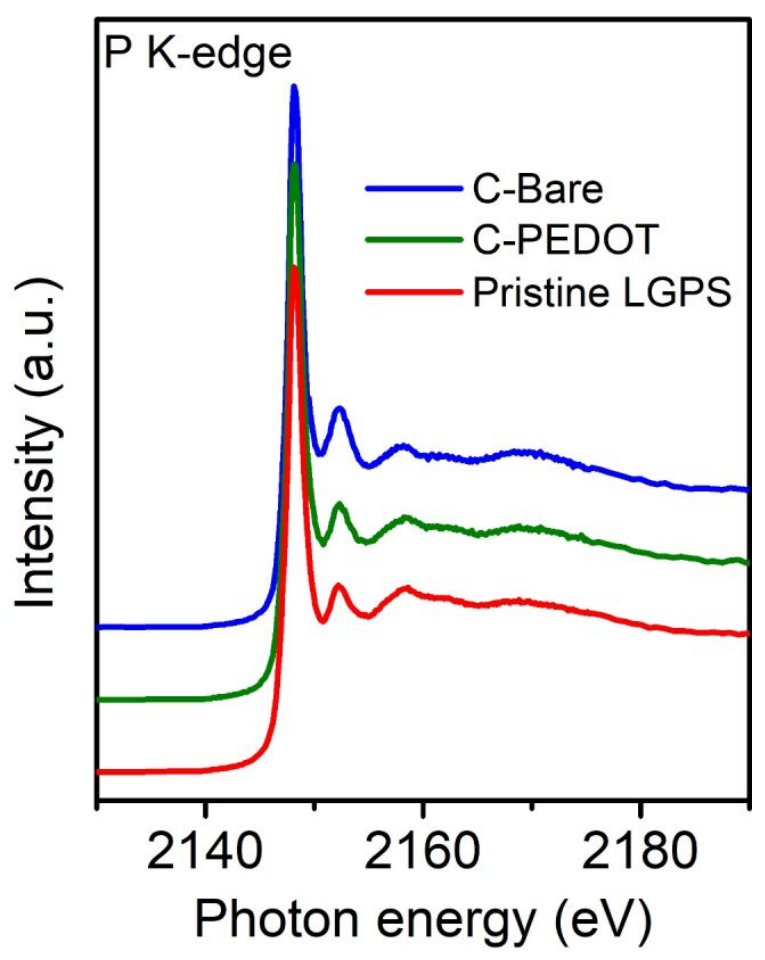

Figure S16. P K-edge XANES spectra of the pristine LGPS, cycled bare electrode (CBare), and cycled PEDOT modified electrode (C-PEDOT). 

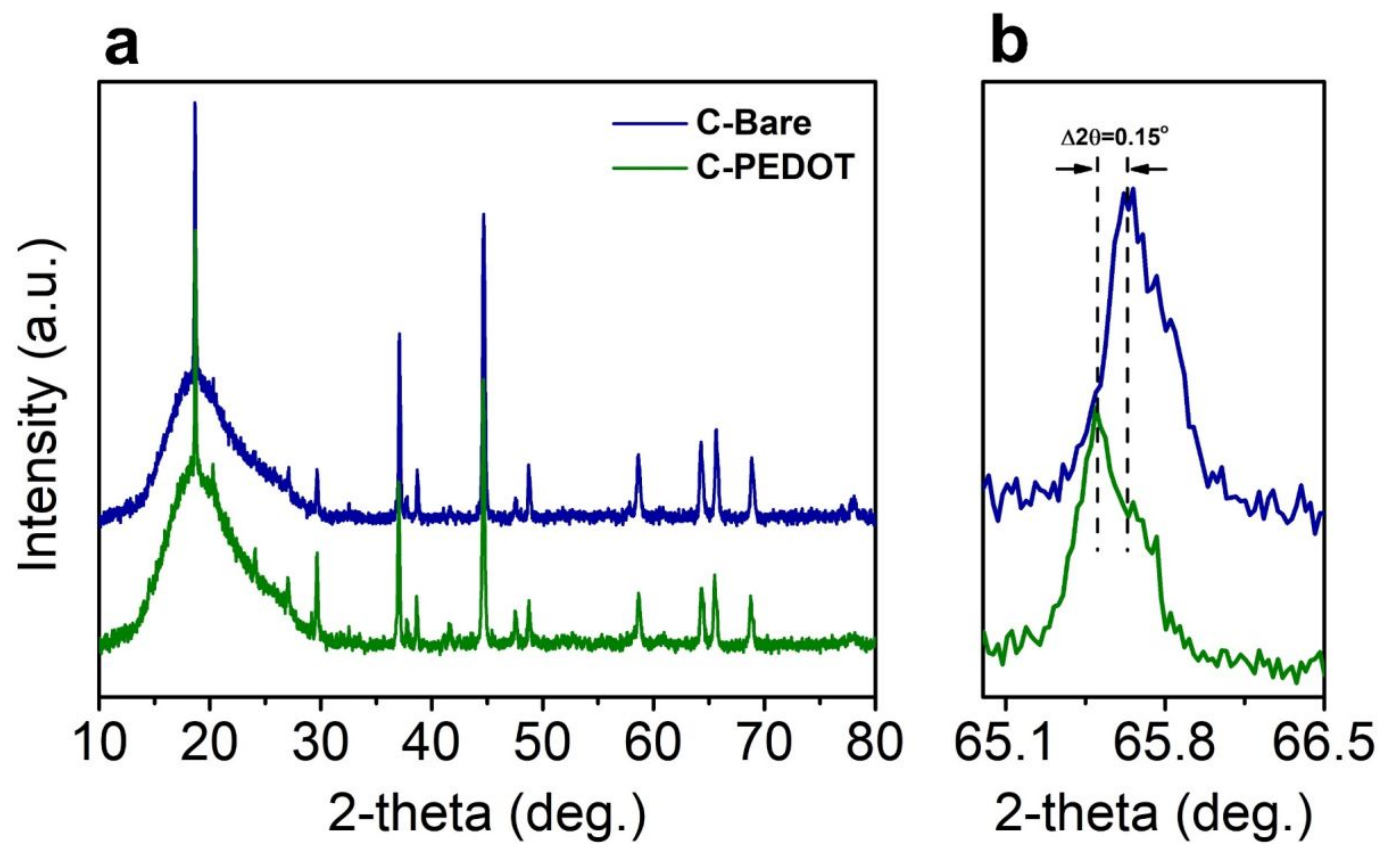

Figure S17. a) XRD patterns and b) the zoom-in patterns of the cycled bare electrode (C-Bare) and cycled PEDOT modified electrode (C-PEDOT). 

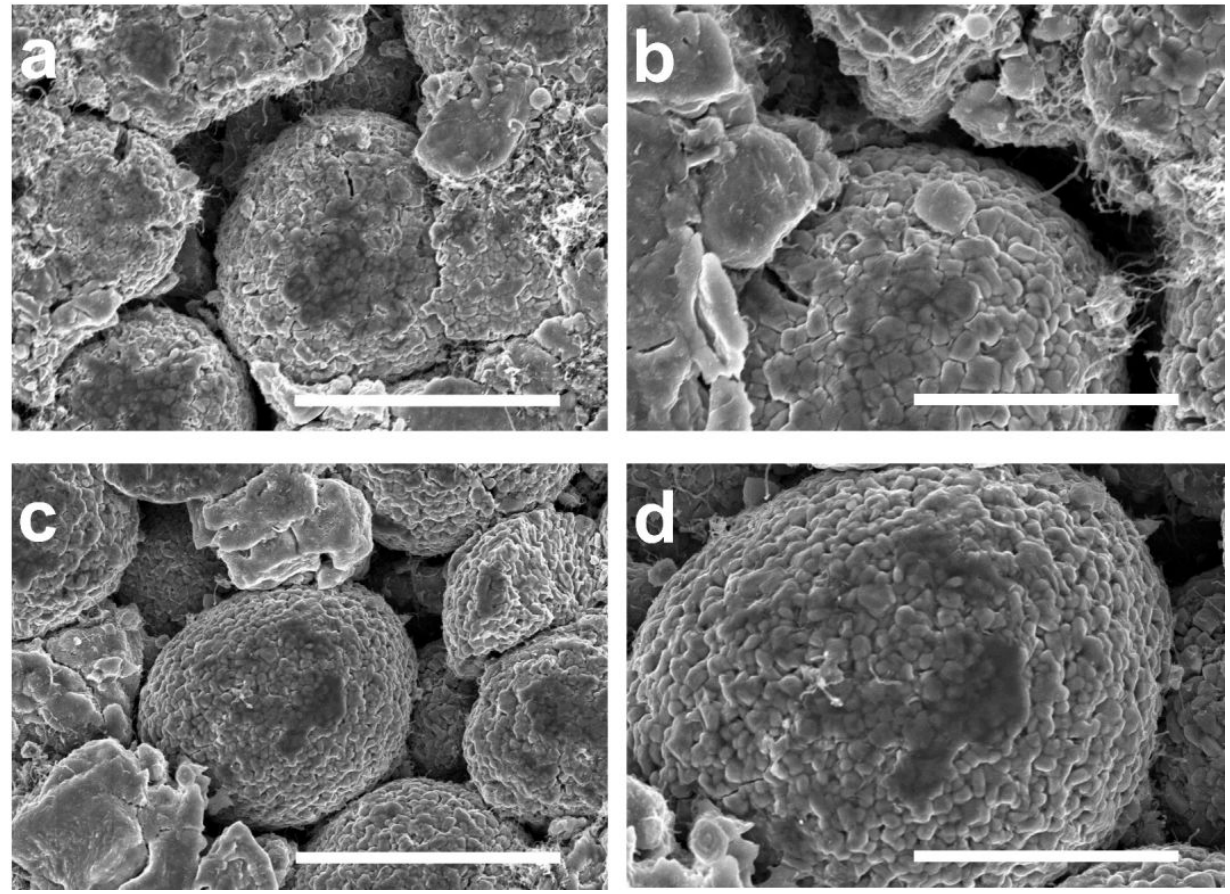

Figure S18. SEM images of a-b) cycled bare electrode; c-d) cycled PEDOT modified electrode. Scale bars in the figures of a and c) $10 \mu \mathrm{m}, \mathrm{b}$ and d) $5 \mu \mathrm{m}$. 

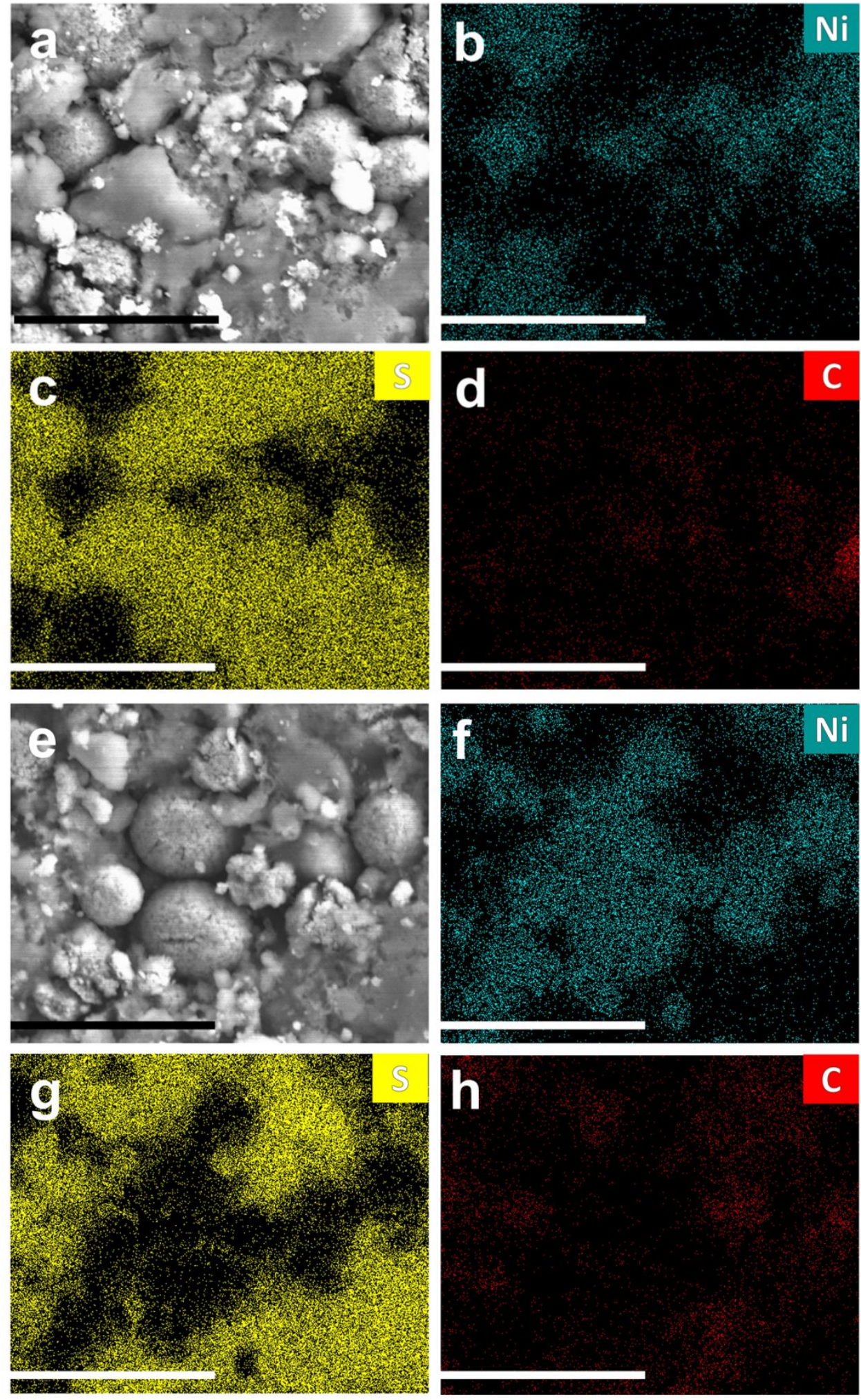

Figure S19. SEM-EDX mappings of the cycled a-d) bare and e-h) PEDOT modified electrodes. $b$ and f) Ni, c and g) S, d and h) C. Scale bars in the figures of $20 \mu \mathrm{m}$. 
Table S1. Resistances obtained from equivalent circuit fitting of EIS experimental data in Figure 3e.

\begin{tabular}{|c|c|c|c|}
\hline Samples & $\mathrm{R} 1(\Omega)$ & $\mathrm{R} 2(\Omega)$ & $\mathrm{R} 3(\Omega)$ \\
\hline Bare & 36 & 1336 & 8506 \\
\hline $2 \mathrm{~nm}$ PEDOT & 29 & 987 & 5140 \\
\hline $5 \mathrm{~nm}$ PEDOT & 26 & 575 & 7705 \\
\hline $10 \mathrm{~nm}$ PEDOT & 40 & 856 & 2202 \\
\hline
\end{tabular}

